\title{
Interdiffusion in the Ir-Rich Solid Solutions of Ir-Pt, Ir-Rh, and Ir-Re Binary Alloys
}

\author{
Nobuaki Sekido, Akinori Hoshino, Masahiro Fukuzaki, Tomohiro Maruko, and Yoko Yamabe-Mitarai
}

\author{
(Submitted November 16, 2010; in revised form February 14, 2011)
}

\begin{abstract}
Interdiffusivity in the Ir-rich solid solutions of $\operatorname{Ir}-\mathrm{X}(\mathrm{X}=\mathrm{Pt}, \mathrm{Rh}$ and $\mathrm{Re})$ binary alloys was investigated in the temperature range of 2073-2373 K. The interdiffusion coefficients within the solute concentration range up to 10 at.\% were evaluated through conventional diffusion-couple experiments. The composition dependence of the interdiffusion coefficient was negligible in the Ir-Rh and Ir-Re solid solutions. On the other hand, some composition dependence was evident in the interdiffusion coefficient of the Ir-Pt solid solution, which increased with increasing Pt content. In comparison with the tracer self-diffusivity of pure Ir, the interdiffusivity in the Ir-Rh and Ir-Pt solid solutions was higher, but that in Ir-Re was lower within the temperature range studied.
\end{abstract}

Keywords Boltzmann-Matano analysis, diffusivity measurements, electron probe microanalysis (EPMA), interdiffusion

\section{Introduction}

Despite the limited resource availability, Ir plays key roles in chemical, electronics, and electrochemical industries. ${ }^{[1]}$ The major advantages of Ir are its high melting point $(2739 \mathrm{~K})$, high corrosion resistance and high elastic moduli (second highest to Os among the pure metals). Because of these benefits, Ir is often employed in the electrodes for the production of chlorine in the chlor-alkali process, crucibles for the growth of scintillator crystals, spark plugs for automotives, extrusion dies for some ceramic fibers, and claddings of radioactive fuel in radioisotope thermoelectric generators used for space power. Beside these applications, Ir compounds are used as catalysts for acetic acid production.

Recently Ir based alloys have attracted considerable interest for potential high temperature structural applications. Previous studies have demonstrated that Ir can exhibit substantially enhanced high temperature strength through optimal alloying strategies. ${ }^{[2-4]}$ Although pure Ir possesses inadequate oxidation resistance (for volatile oxides $\mathrm{IrO}_{3}$ forms upon air exposure at above $1293 \mathrm{~K}^{[5-7]}$ ), this can be improved by the addition of $\mathrm{Pt}$ or $\mathrm{Rh} .{ }^{[8]}$

Another prospective field of $\mathrm{Ir}$ application is as the coating for high temperature materials. In modern turbine engines, thermal barrier coatings (TBCs) are comprised of a

Nobuaki Sekido and Yoko Yamabe-Mitarai, National Institute for Materials Science, 1-2-1 Sengen, Tsukuba, Ibaraki 305-0047, Japan; Akinori Hoshino, Masahiro Fukuzaki, and Tomohiro Maruko, Furuya Metal Co., Ltd., 1915 Morisoejima, Chikusei, Ibaraki 308-0861, Japan. Contact e-mails: sekido.nobuaki@nims.go.jp, hoshino@furuya metals.co.jp, fukuzaki@furuyametals.co.jp, maruko@furuyametals. co.jp and MITARAI.Yoko@nims.go.jp ceramic topcoat and an underlying bond-coat and are applied on the surface of Ni-base superalloys. Commonly, yttria-stabilized zirconia (YSZ) is employed in the topcoat, and either a metallic overlay coating with an MCrAlY composition $(\mathrm{M}=\mathrm{Ni}, \mathrm{Co})$, an aluminide diffusion coating, or a noble element modified aluminide coating (mainly $\mathrm{PtAl}$ ) is used as the bond-coat. It has been demonstrated that Ir addition is effective in enhancing the oxidation and hot corrosion resistance of PtAl. ${ }^{[9-12]}$ For further improvement in protection performances, application of Ir based alloys to the bond-coats has been proposed. ${ }^{[13-19]}$ At the same time, a Re modified aluminide coating has been shown to exhibit notable performance as a diffusion barrier. ${ }^{[20]}$

Despite such promising performances of Ir in structural and coating application fields, only limited information is available for atomic diffusivity in Ir based alloys. ${ }^{[21,22]}$ Thus in this study, the interdiffusion in the Ir-rich solid solutions of Ir-Pt, Ir-Rh and Ir-Re binary alloys is investigated. Ir forms continuous FCC solid solutions with both $\mathrm{Pt}$ and $\mathrm{Rh}$ across the entire composition range of $0-100 \%$ solute. Re in contrast to the FCC structures of Pt \& Rh crystallizes with the HCP structure. However, the Ir-rich terminal solution is FCC in nature, and a somewhat speculative phase diagram $^{[23]}$ shows this solution extending to a peritectic decomposition at $\sim 35$ at. $\%$ Re. Thus all solution starting at 10 at.\% solute which were used in this investigation had an FCC structure. The interdiffusion coefficients were determined by conventional diffusion-couple experiments at temperatures from 2073 to $2373 \mathrm{~K}$.

\section{Experimental Procedures}

Alloys with the nominal compositions of Ir-10 at.\%Pt, Ir-10 at. $\% \mathrm{Rh}$, and $\mathrm{Ir}-10$ at. $\%$ Re were prepared by arcmelting high purity raw materials under an Ar atmosphere. The homogenized alloys and pure-Ir were mechanically polished and diffusion-bonded at $1773 \mathrm{~K}$ for 30 min under vacuum at the compressive stress of $40 \mathrm{MPa}$. Hereafter, 
three types of diffusion couples that are comprised of pure-Ir and an Ir-X alloy (X $=\mathrm{Pt}, \mathrm{Rh}$ and $\mathrm{Re}$ ) are denoted as $\mathrm{Ir} / \mathrm{Ir}-$ $10 \mathrm{Pt}, \mathrm{Ir} / \mathrm{Ir}-10 \mathrm{Rh}$, and $\mathrm{Ir} / \mathrm{Ir}-10 \mathrm{Re}$, respectively. These diffusion couples were annealed at 2073, 2173, 2273, and $2373 \mathrm{~K}$ under vacuum $\left(<10^{-3} \mathrm{~Pa}\right)$. Concentration profiles across the bonded interface were measured by a field emission electron probe micro-analyzer (FE-EPMA: JEOL JXA8900R) at the acceleration voltage of $20 \mathrm{kV}$ with the beam current of $100 \mathrm{nA}$ and a probe size smaller than $1 \mu \mathrm{m}$. The characteristic x-rays of Ir-L $\alpha$, Pt-L $\alpha$, Rh-L $\alpha$, Re-L $\alpha$ were used for the measurements. Two concentration profiles were measured in each diffusion couple.

\section{Results}

\subsection{Analysis Technique}

A diffusion couple comprised of a pair of semi-infinite solids is considered. If the interdiffusion coefficient $\tilde{D}$ is constant, the solute concentration $c$ at position $x$ after annealing time $t$ is given by

$c(x, t)=\frac{c_{1}+c_{0}}{2}+\frac{c_{1}-c_{0}}{2} \operatorname{erf}\left(\frac{x-x_{0}}{2 \sqrt{\tilde{D} t}}\right)$

where $x_{0}$ is the coordinate center, and $c_{0}, c_{1}$ were the terminal compositions of the diffusion couple. By rearranging Eq 1, we here define $q(x, t)$ as

$\frac{x-x_{0}}{2 \sqrt{\tilde{D} t}}=\operatorname{erf}^{-1}\left(\frac{2 c(x, t)-c_{1}-c_{0}}{c_{1}-c_{0}}\right) \equiv q(x, t)$

If an experimentally measured concentration profile follows $\mathrm{Eq} 1$ and $q$ calculated from $c$ by $\mathrm{Eq} 2$ is plotted against $x$, the plot must show a straight line. This type of plot is often called "probability plot", and the interdiffusion coefficient can be determined from the slope of the regression line, which is equal to $1 /\left(2(\tilde{D} t)^{1 / 2}\right)$.

This, in turn, means that, if the probability plot shows a curved nature, the concentration profile does not follow Eq 1; i.e., some compositional dependence is present in the interdiffusion coefficients. In such a case, the BoltzmannMatano method ${ }^{[24]}$ can be applied. It has been demonstrated that, by the introduction of the Boltzmann parameter $\lambda$, a nonlinear partial differential equation of Fick's second law can be transformed into an ordinary differential equation as follows:

$\lambda=\frac{x-x_{\mathrm{M}}}{\sqrt{t}}$

$-\frac{\lambda}{2} \frac{d c}{d \lambda}=\frac{d}{d \lambda}\left(D \frac{d c}{d \lambda}\right)$

where $x_{M}$ is the position of the Matano plane that satisfies:

$\int_{C 0}^{C 1}\left(x-x_{\mathrm{M}}\right) d c=0$
By solving Eq 4 with the geometric requirements of the diffusion couple as the boundary condition, ${ }^{[25-27]}$ the interdiffusion coefficient at solute concentration $c^{*}$ can be determined by analyzing an experimentally obtained concentration profile with the following equation.

$\tilde{D}\left(c^{*}\right)=-\frac{1}{2 t}\left(\frac{d x}{d c}\right)_{C^{*}} \int_{C^{0}}^{C^{*}}\left(x-x_{M}\right) d c$

The above analysis techniques premise that, regardless of the presence of concentration dependence in the interdiffusion coefficients, the solute concentration profile is expressed by a function of the Boltzmann parameter that combines time and distance as a single variable. In other words, the concentration profiles of diffusion couples annealed for different periods should be identical when the diffusion distance is normalized by $t^{1 / 2}$. An example is shown in Fig. 1, where the Re concentrations in the $\mathrm{Ir} / \mathrm{Ir}$ $10 \mathrm{Re}$ diffusion couples annealed for 72 and $240 \mathrm{~h}$ at $2273 \mathrm{~K}$ are plotted against the Boltzmann parameter. Two concentration profiles reasonably agree with each other, suggesting that the above techniques are valid to apply for analyzing the interdiffusion coefficients in this study.

\subsection{Determination of Interdiffusion Coefficients}

It was confirmed by SEM and EPMA examinations that the bonding treatment yielded well-bonded planar interfaces with marginal diffusion layers for all the diffusion couples. After diffusion-annealing, interdiffusion resulted in the development of planar diffusion layers with thickness enough to analyze the interdiffusion coefficients. No Kirkendall voids were observed near the interfaces for all the diffusion couples.

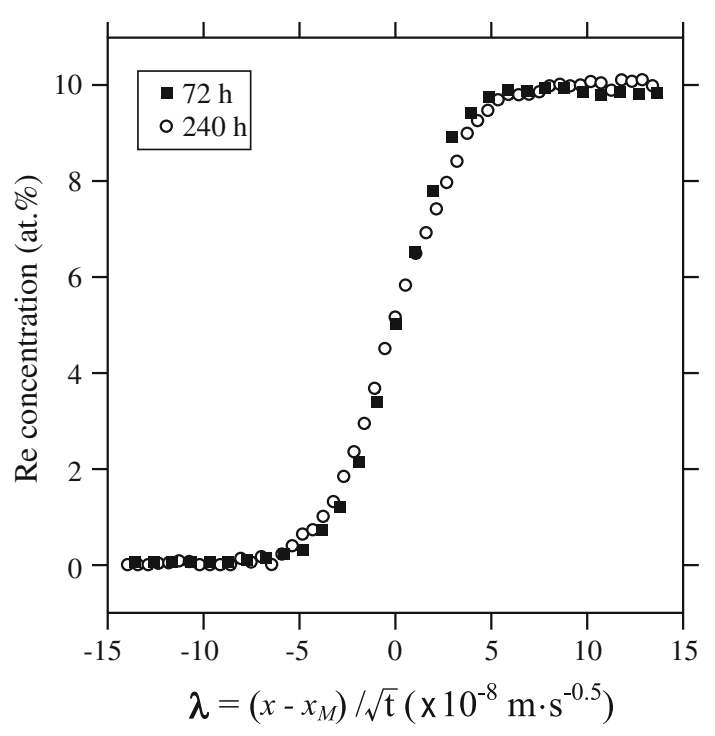

Fig. 1 Re concentration profiles in the interdiffusion zones of $\mathrm{Ir} / \mathrm{Ir}-10 \mathrm{Re}$ diffusion couples annealed at $2273 \mathrm{~K}$ for 72 and $240 \mathrm{~h}$. Two concentration profiles are plotted against the Boltzmann parameter, $\lambda$ 
Figure 2 shows the concentration profiles obtained from (a) $\mathrm{Ir} / \mathrm{Ir}-10 \mathrm{Rh}$ and (b) $\mathrm{Ir} / \mathrm{Ir}-10 \mathrm{Re}$ diffusion couples annealed at $2273 \mathrm{~K}$ for $72 \mathrm{~h}$. To ascertain the presence of concentration-dependence in the interdiffusion coefficients, these profiles are assessed by the probability plot as in Fig. 2(c) for $\mathrm{Ir} / \mathrm{Ir}-10 \mathrm{Rh}$ and (d) for $\mathrm{Ir} / \mathrm{Ir}-10 \mathrm{Re}$. Within the range of $-1 \leq q \leq 1$, which covers about $85 \%$ of the concentration windows, the plots can be fit by straight lines for both $\mathrm{Ir} / \mathrm{Ir}-$ $10 \mathrm{Rh}$ and $\mathrm{Ir} / \mathrm{Ir}-10 \mathrm{Re}$. This suggests that the interdiffusion coefficients can be regarded to be constant within this composition range, and thus the interdiffusion coefficient is simply calculated from the slope of the regression lines in the probability plot, which is equal to $1 /\left(2(\tilde{D} t)^{1 / 2}\right)$.

By examining the concentration profiles at 2073, 2173, and $2373 \mathrm{~K}$, it is found that the interdiffusion coefficients in the Ir-Rh and Ir-Re solid solutions can be regarded to be constant within the ranges of compositions and temperatures. Thus, the same procedures have been applied for the determination of the interdiffusion coefficients, of which results are summarized in Table 1 .

Figure 3 shows a concentration profile and its probability plot for the Ir/Ir-10Pt diffusion couple annealed at $2273 \mathrm{~K}$
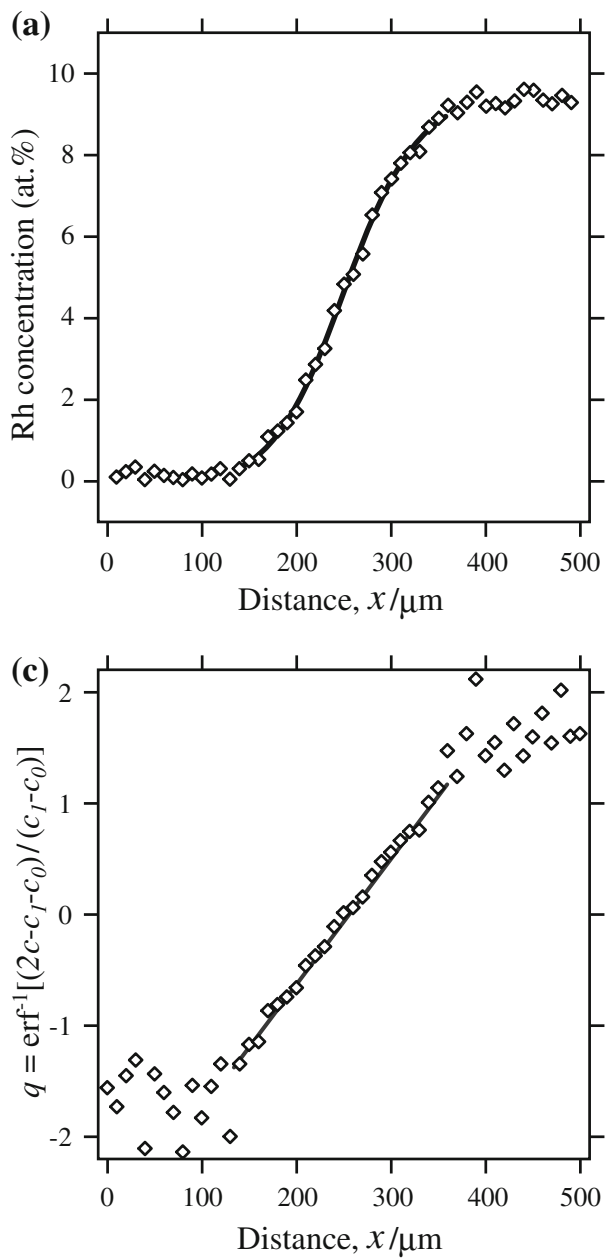

for $72 \mathrm{~h}$. The probability plot appears to be slightly curved, suggesting that the interdiffusion coefficient shows some compositional dependence within this compositional range. The probability plot in Fig. 3(b) is approximated by the

Table 1 Interdiffusion coefficients in determined in this study

\begin{tabular}{|c|c|c|c|}
\hline \multirow{2}{*}{$\begin{array}{l}\text { Temperature } \\
T, \mathbf{K}\end{array}$} & \multirow{2}{*}{$\begin{array}{c}\text { Time } \\
t, \mathbf{h}\end{array}$} & \multicolumn{2}{|c|}{ Interdiffusion coefficient $\tilde{D}, \mathrm{~m}^{2} / \mathrm{s}$} \\
\hline & & $\operatorname{Ir} / \mathbf{I r}-10 \operatorname{Re}$ & Ir/Ir-10Rh \\
\hline \multirow[t]{2}{*}{2073} & 336 & $4.30 \times 10^{-17}$ & $4.36 \times 10^{-16}$ \\
\hline & & $3.32 \times 10^{-17}$ & $4.30 \times 10^{-16}$ \\
\hline \multirow[t]{2}{*}{2173} & 168 & $1.43 \times 10^{-16}$ & $1.91 \times 10^{-15}$ \\
\hline & & $8.19 \times 10^{-17}$ & $1.88 \times 10^{-15}$ \\
\hline \multirow[t]{4}{*}{2273} & 72 & $2.67 \times 10^{-16}$ & $7.55 \times 10^{-15}$ \\
\hline & & $3.37 \times 10^{-16}$ & $7.25 \times 10^{-15}$ \\
\hline & 240 & $4.89 \times 10^{-16}$ & \\
\hline & & $4.68 \times 10^{-16}$ & \\
\hline \multirow[t]{2}{*}{2373} & 24 & $9.75 \times 10^{-16}$ & $1.97 \times 10^{-14}$ \\
\hline & & $1.29 \times 10^{-15}$ & $2.11 \times 10^{-14}$ \\
\hline
\end{tabular}
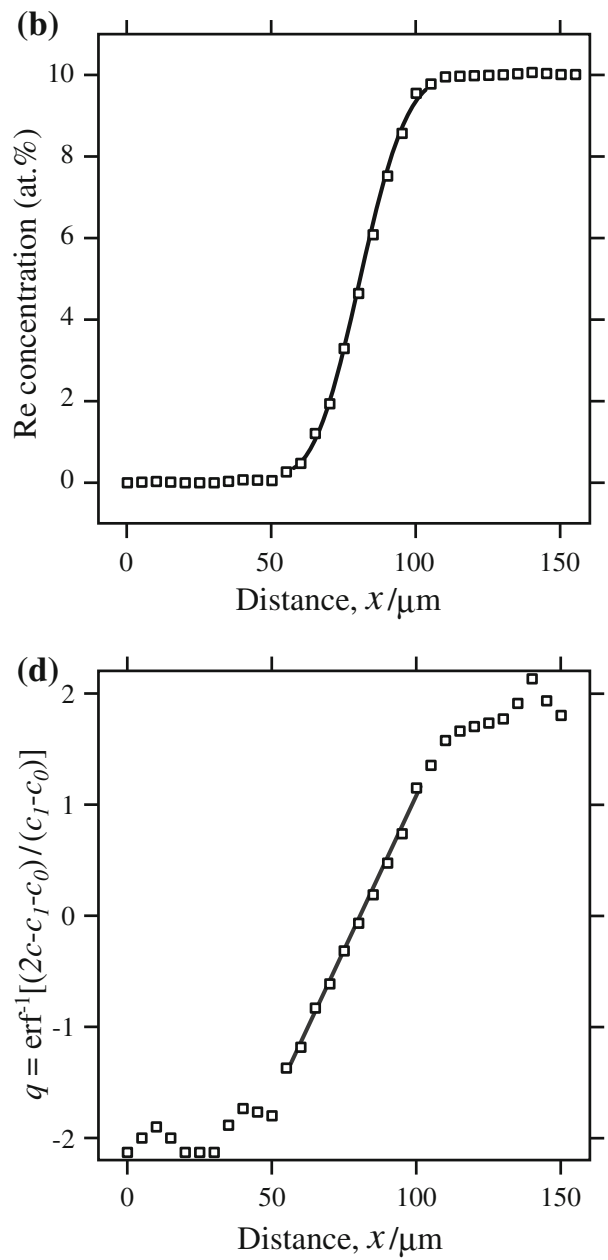

Fig. 2 Concentration profiles and their probability plots in (a, c) Ir/Ir-10Rh and (b, d) Ir/Ir-10Re diffusion couples annealed at $2273 \mathrm{~K}$ for $72 \mathrm{~h}$. The solid curves in $(\mathrm{a}, \mathrm{b})$ represent the results of back-transformation from the regression lines in the probability plots (c, d) 

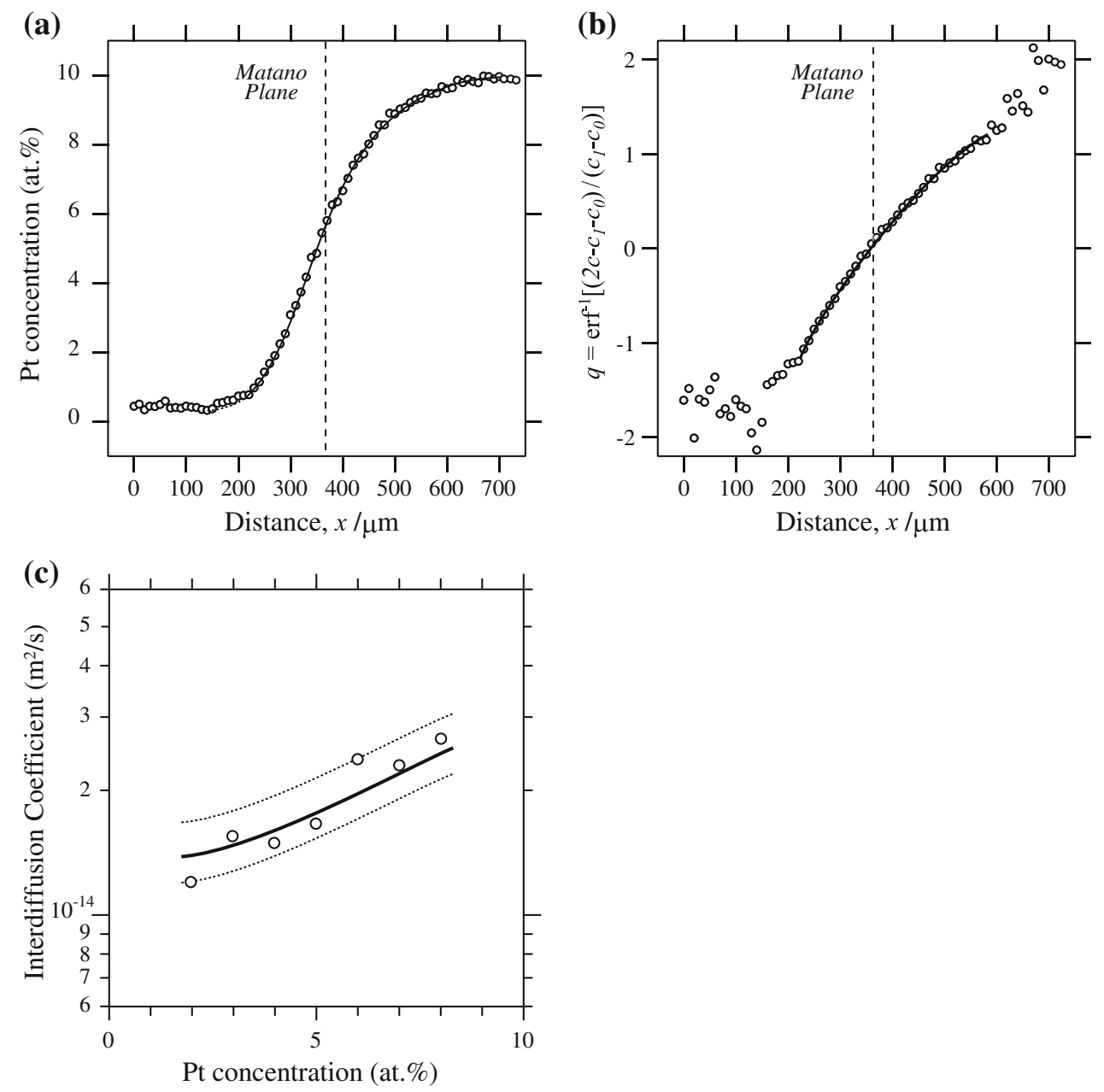

Fig. 3 (a) Pt concentration profile and (b) its probability plot for an Ir/Ir-10Pt diffusion couple annealed at $2273 \mathrm{~K}$ for $72 \mathrm{~h}$. (c) The interdiffusion coefficients at $2273 \mathrm{~K}$ against Pt content. The solid line in (c) represents the results of the B-M analysis performed on the regression curve, while the open circles on raw data. The broken lines show the range of errors

third order of polynomial within the range of $-1.2 \leq q \leq 1.2$, which covers more than $90 \%$ of the whole concentration range of the diffusion couple. The regression curve is backtransformed into the original coordinates as drawn by the solid line in Fig. 3(a). Here, the back-transformation is also made on the outside of the regression range, i.e., near the terminal compositions. It is seen that the profile is well reproduced for all over the range of compositions in the diffusion couple.

For the determination of the interdiffusion coefficients in this study, the Boltzmann-Matano (B-M) analysis was performed on the regression curves that were deduced from the probability plots. Figure 3(c) shows the result of the B$\mathrm{M}$ analysis on the profile in Fig. 3(a). We also performed the $\mathrm{B}-\mathrm{M}$ analyses on the raw data to define the probable range of errors, which is depicted by open circles and broken lines in Fig. 3(c). It is noted that only the results at the Pt concentrations from 2 to 8 at.\% are shown in Fig. 3(c), since the B-M analysis often gives large errors at the end of the diffusion profile. ${ }^{[26]}$
The same procedures have been applied for the determination of the interdiffusion coefficients at 2073, 2173, and $2373 \mathrm{~K}$. The variation of the interdiffusion coefficients against the $\mathrm{Pt}$ content is summarized in Fig. 4. The interdiffusion coefficient at 2 at.\% Pt is about a factor of two higher than that at 8 at.\% $\mathrm{Pt}$ in this temperature range.

\subsection{Temperature Dependence of Interdiffusivity}

Figure 5 shows the Arrhenius plots for the interdiffusion coefficients in the fcc solid solutions of Ir-Re, Ir-Rh, and Ir-Pt binary alloys. For Ir-Pt, the data at 5 at. $\%$ is shown as the representative of the compositional dependant coefficients. The tracer self-diffusion coefficient of pure $\operatorname{Ir}^{[28]}$ is also shown for comparison. The interdiffusivity in the Ir-Rh and Ir-Pt solid solutions is higher than the tracer self-diffusivity of pure Ir. On the other hand, the interdiffusivity in the Ir-Re solid solution is lower than the self-diffusivity of pure Ir. The Arrhenius plots in Fig. 5 clearly show a linear correlation between the logarithm of interdiffusion coefficients and 


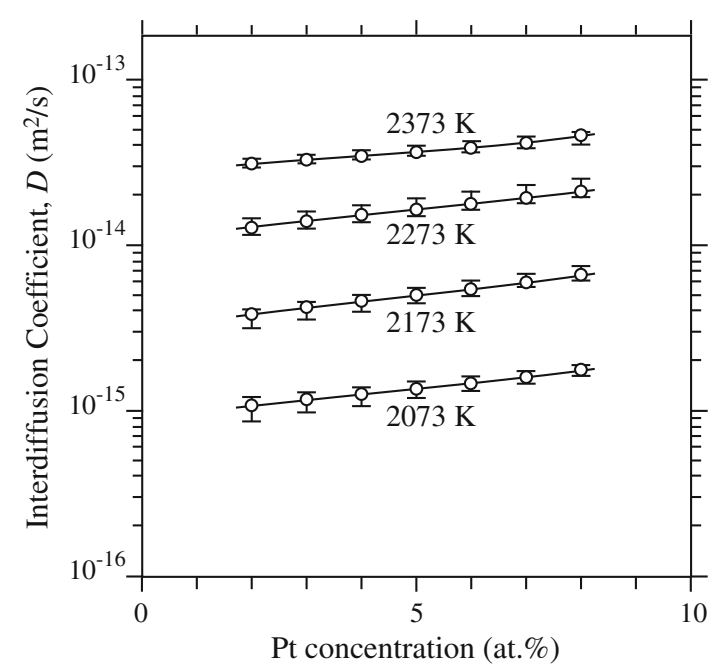

Fig. 4 The interdiffusion coefficients in the Ir-Pt solid solution at $2073,2173,2273$, and $2373 \mathrm{~K}$

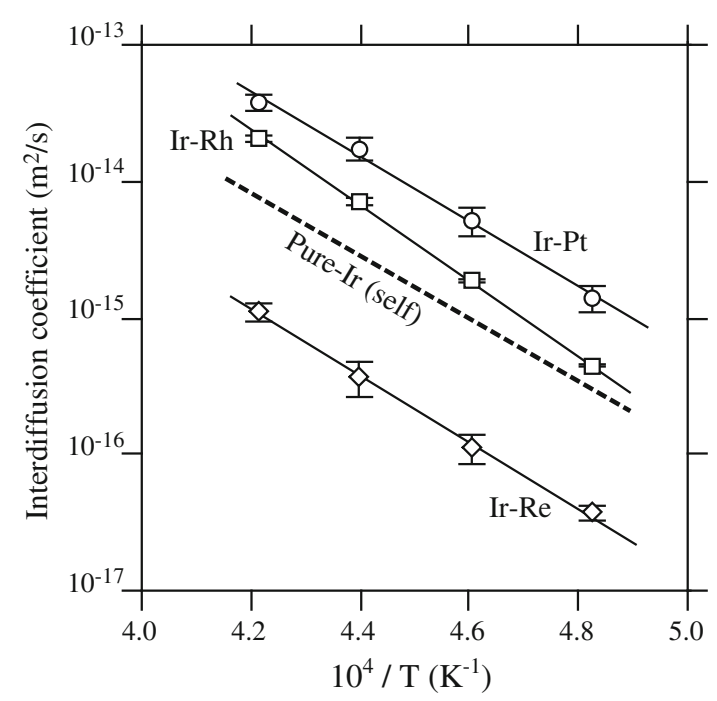

Fig. 5 Arrhenius plots of the interdiffusion coefficients in the Ir-Re, Ir-Rh, and Ir-Pt solid solutions. The broken line represents the tracer self-diffusion coefficient of pure $\operatorname{Ir}^{[28]}$

the inverse temperature, suggesting that the diffusion coefficients follow the Arrhenius law expressed by:

$\tilde{D}=D_{0} \exp \left(-\frac{Q}{R T}\right)$

where $D_{0}$ is a prefactor, $Q$ an apparent activation energy, $R$ gas constant, and $T$ absolute temperature. Table 2 summarizes the values of $D_{0}$ and $Q$ determined in this study. It is found that the apparent activation energies for interdiffusion in the Ir-Pt, Ir-Rh and Ir-Re solid solutions are higher than that of the self-diffusion of pure Ir within the studied composition range. It is also seen that the apparent activation energy for interdiffusion in the Ir-Pt solid solution tends to decrease with increasing Pt content.
Table 2 Activation energies and prefactors for the interdiffusion coefficients

\begin{tabular}{lcc}
\hline & \multicolumn{2}{c}{$\tilde{\boldsymbol{D}}$} \\
\cline { 2 - 3 } Composition & $\boldsymbol{\operatorname { l o g }}_{\mathbf{1 0}}\left(\boldsymbol{D}_{\mathbf{0}}, \mathbf{~ m}^{\mathbf{2}} / \mathbf{s}\right)$ & $\boldsymbol{Q}, \mathbf{~ k J} / \mathbf{m o l}$ \\
\hline$\sim 10$ at.\% Re & $-4.9 \pm 1.0$ & $456 \pm 36$ \\
$\sim 10$ at.\% Rh & $-2.0 \pm 0.1$ & $529 \pm 5$ \\
2 at.\% Pt & $-3.3 \pm 1.0$ & $462 \pm 45$ \\
4 at.\% Pt & $-3.4 \pm 0.8$ & $456 \pm 33$ \\
6 at.\% Pt & $-3.4 \pm 0.6$ & $451 \pm 24$ \\
8 at.\% Pt & $-3.4 \pm 0.7$ & $449 \pm 29$ \\
Ir (self) & -4.44 & 439 \\
\hline
\end{tabular}

\section{Discussions}

Upon analysis of the interdiffusion coefficients, the change in partial molar volumes is neglected in this study. Although an analytic methodology that takes molar volume change into account has been proposed by Sauer and Freise, ${ }^{[29]}$ it is pointed out that their method yields substantially the same result that can be obtained by the Boltzmann-Matano analysis, when the partial molar volume change is smaller than $1 \% .{ }^{[26]}$ Based on the reported lattice parameters of Ir, Pt and their alloys, ${ }^{[30-32]}$ the variation in the molar volume within the diffusion couples can be estimated to be less than $0.3 \%$ at room temperature and at $1973 \mathrm{~K}$. To our best knowledge, information on the lattice parameters, as well as the partial molar volumes of Ir-Rh and Ir-Re binary alloys is not available. Still, it is believed that the variation of the molar volumes in $\mathrm{Ir} / \mathrm{Ir}-10 \mathrm{Rh}$ and $\mathrm{Ir} / \mathrm{Ir}-10 \mathrm{Re}$ diffusion couples would be comparable to that in Ir/Ir-10Pt, since their Goldschmidt atomic radii are not largely different; Ir: $0.135 \mathrm{~nm}, \mathrm{Pt}: 0.138 \mathrm{~nm}, \mathrm{Rh}: 0.134 \mathrm{~nm}$, and Re: $0.138 \mathrm{~nm}^{[33]}$

In comparison with the tracer self-diffusivity of pure Ir, the interdiffusivity in the Ir-Rh and Ir-Pt solid solutions was higher, but that in Ir-Re was lower within the temperature range studied. According to the binary phase diagrams, ${ }^{[23]}$ the addition of $\mathrm{Pt}$ and $\mathrm{Rh}$ to pure-Ir lowers the melting temperature, while Re addition raises the melting temperature. This trend agrees with the empirical knowledge: at a given temperature, interdiffusivity increases if second elemental addition lowers the melting temperature of the solvent, and decreases if the solute raises the melting temperature. ${ }^{[25]}$

Although Pt addition lowers but Re addition raises the melting temperature of Ir, their apparent activation energies for interdiffusion are comparable. Instead, the apparent activation energy for interdiffusion in the Ir-Rh solid solution seems to be higher than that for the Ir self-diffusion and interdiffusion in the Ir-Pt and Ir-Re solid solutions. It has been documented that the apparent activation energy for interdiffusion of platinum-group metals (PGMs) in Ni tends to increase with decreasing misfit, where the misfit is defined as the difference in the Goldschmidt atomic radii between Ni and PGMs. ${ }^{[34]}$ They explained this trend by the solute-vacancy binding energy, which increases with 
increasing misfit. The same tendency is observed for the interdiffusion in the Ir-rich solid solutions of Ir-Pt, Ir-Rh, and Ir-Re alloys. As noted above, $\mathrm{Rh}$ has the smallest misfit in the atomic radii against Ir, and shows the highest apparent activation energy for interdiffusion. It should be noted that this is only a qualitative explanation for the experimental results in this study. In fact, the previous study ${ }^{[21]}$ has demonstrated that the apparent activation energy for the interdiffusion in Ir-Nb solid solution is $496( \pm 15) \mathrm{kJ} / \mathrm{mol}$, which is higher than that of $\mathrm{Pt}$ or $\mathrm{Re}$, although the Goldschmidt atomic radius of $\mathrm{Nb}(0.147 \mathrm{~nm})^{[33]}$ is larger than that of $\mathrm{Pt}$ or Re. More detailed studies are required to identify the factors that contribute to the apparent activation energies for interdiffusion.

\section{Conclusions}

Interdiffusion in the Ir-Pt, Ir-Rh, and Ir-Re solid solutions has been studied. Within the solute composition range up to $\sim 10$ at. $\%$, the interdiffusion coefficients in the Ir-Rh, and IrRe solid solutions show negligible change with composition. On the other hand, some composition dependence is evident in the interdiffusion coefficient of the Ir-Pt solid solution phase. The apparent activation energy of diffusion in the Ir-Pt solid solution decreases with increasing $\mathrm{Pt}$ content. Within the temperature range of 2073-2373 K, the interdiffusion coefficients in the fcc solid solutions of Ir-Re, Ir-Rh, and Ir-Pt binary alloys are expressed by the Arrhenius-type formula as follows.

$$
\begin{aligned}
& \tilde{D}=10^{-4.8 \pm 1.4} \exp \left(-\frac{460 \pm 58[\mathrm{~kJ} / \mathrm{mol}]}{R T}\right)\left[\mathrm{m}^{2} / \mathrm{s}\right], \\
& \text { for } \sim 10 \text { at. } \% \mathrm{Re} \\
& \tilde{D}=10^{-2.0 \pm 0.1} \exp \left(-\frac{529 \pm 5[\mathrm{~kJ} / \mathrm{mol}]}{R T}\right)\left[\mathrm{m}^{2} / \mathrm{s}\right], \\
& \text { for } \sim 10 \text { at. } \% \mathrm{Rh} \\
& \tilde{D}=10^{-3.4 \pm 0.7} \exp \left(-\frac{453 \pm 28[\mathrm{~kJ} / \mathrm{mol}]}{R T}\right)\left[\mathrm{m}^{2} / \mathrm{s}\right] \\
& \text { for } 5 \text { at. } \% \mathrm{Pt}
\end{aligned}
$$

In comparison with the tracer self-diffusivity of pure Ir, the interdiffusivity in the Ir-Rh and Ir-Pt solid solutions was higher, but that in Ir-Re was lower within the temperature range studied. This trend is qualitatively explained by the change in the melting temperature by second elemental additions.

\section{Acknowledgment}

Assistance with EPMA from Ms. Yuko Isozaki (NIMS) is gratefully acknowledged.

\section{References}

1. D. Jollie, Platinum 2009, Johnson-Matthey PLC, www. platinum.matthey.com/publications/

2. Y. Yamabe-Mitarai, Y. Gu, C. Huang, R. Volkl, and H. Harada, Platinum-Group-Metal-Based Intermetallics as High-Temperature Structural Materials, JOM, 2004, 56(9), p 34-39

3. Y. Yamabe-Mitarai, T. Maruko, T. Miyazawa, and T. Morino, Solid Solution Hardening Effect of Ir, Mater. Sci. Forum, 2005, 475-479, p 703-706

4. Y. Yamabe-Mitarai, Y. Ro, T. Maruko, and H. Harada, Ir-Base Refractory Superalloys for Ultrahigh Temperatures, Metall. Mater. Trans. A, 1998, 29, p 537-549

5. C.A. Krier and R.I. Jaffee, Oxidation of the Platinum-Group Metals, J. Less-Common Met., 1963, 5, p 411-431

6. J.C. Chaston, Reactions of Oxygen with the Platinum Metals, Platinum Met. Rev., 1965, 9, p 51-56

7. N. Sekido, H. Murakami, and Y. Yamabe-Mitarai, Phase Equilibria and Oxidation Behavior of Ir-rich Ir-Y Binary Alloys, J. Alloys Compd., 2009, 476, p 107-112

8. R.G. Waltenberg, H.H. Edwin, and B. Bert, Alloy, U.S. Patent $1,850,819,1932$

9. G. Fisher, P.K. Datta, and J.S. Burnell-Gray, An Assessment of the Oxidation Resistance of an Iridium and an Iridium/ Platinum Low-Activity Aluminide/MarM002 System at $1100{ }^{\circ} \mathrm{C}$, Surf. Coat. Technol., 1999, 113, p 259-267

10. Y. Wu, A. Suzuki, H. Murakami, and S. Kuroda, Characterization of Electroplated Platinum-Iridium Alloys on the Nickel-base Single Crystal Superalloy, Mater. Trans., 2005, 46, p 2176-2179

11. A. Suzuki, Y. Wu, A. Yamaguchi, H. Murakami, and C.M.F. Rae, Oxidation Behavior of Pt-Ir Modified Aluminized Coatings on Ni-base Single Crystal Superalloy TMS-82+, Oxid. Met., 2007, 68, p 53-64

12. Y.N. Wu, A. Yamaguchi, H. Murakami, and S. Kuroda, Role of Iridium in Hot Corrosion Resistance of Pt-Ir Modified Aluminide Coatings with $\mathrm{Na}_{2} \mathrm{SO}_{4}-\mathrm{NaCl}$ Salt at $1173 \mathrm{~K}$, Mater. Trans., 2006, 47, p 1918-1921

13. K.N. Lee and W.L. Worrell, The Oxidation of IridiumAluminum and Iridium-Hafnium Intermetallics at Temperatures above $1550{ }^{\circ} \mathrm{C}$, Oxid. Met., 1989, 32, p 357-369

14. H. Hosoda, S. Miyazaki, and S. Hanada, Potential of IrAl Base Alloys as Ulatrahigh Temperature Smart Coatings, Intermetallics, 2000, 8, p 1081-1090

15. F. Wu, H. Murakami, and A. Suzuki, Development of an Iridium-Tantalum Modified Aluminide Coating as a Diffusion Barrier on Nickel-base Single Crystal Superalloy TMS-75, Surf. Coat. Technol., 2003, 168, p 62-69

16. H. Murakami, A. Suzuki, F. Wu, P. Kuppusami, and H. Harada, Application of Ir-base Alloys to Novel Oxidation Resistant Bond Coatings, Superalloys-2004, K.A. Green, T.M. Pollock, H. Harada, T.E. Howson, R.C. Reed, J.J. Schirra, and S. Walston, Ed., Sep 19-23, 2004 (Champion, PA), TMS, 2004, p 589-596

17. P. Kuppusami, H. Murakami, and T. Ohmura, Behaviour of Ir24Ta Films on Ni Based Single Crystal Superalloys, Surf. Eng., 2005, 21, p 53-59

18. P. Kuppusami and H. Murakami, Effect of Ta on Microstructure and Phase Distribution in Cyclic Oxidized Ir-Ta Modified Aluminide Coatings on Nickel Base Single Crystal Superalloy, Mater. Sci. Eng. A, 2007, 452-453, p 254-261

19. A. Yamaguchi, H. Murakami, S. Kuroda, and H. Imai, Effect of Hf Addition on Oxidation Properties of Pt-Ir Modified Aluminide Coating, Mater. Trans., 2007, 48, p 2422-2426

20. T. Narita, F. Lang, K.Z. Thosin, T. Yoshioka, T. Izumi, H. Yakuwa, and S. Hayashi, Oxidation Behavior of Nickel-base 
Single-Crystal Superalloy with Rhenium-base Diffusion Barrier Coating System at $1423 \mathrm{~K}$ in Air, Oxid. Met., 2007, 68, p 343-363

21. H. Numakura, T. Watanabe, M. Uchida, Y. Yamabe-Mitarai, and E. Bannai, Chemical Diffusion in the Iridium-rich A1 and $\mathrm{L}_{2}$ Phases in the Ir-Nb System, J. Phase Equilib. Diffus., 2006, 27, p 638-643

22. M. Uchida, H. Numakura, Y. Yamabe-Mitarai, and E. Bannai, Chemical Diffusion in $\mathrm{Ir}_{3} \mathrm{Nb}$, Scripta Mater., 2005, 52, p 11-15

23. H. Okamoto, Desk Handbook-Phase Diagrams for Binary Alloys, ASM International, Materials Park, PA, 2000

24. C. Matano, On the Relation between the Diffusion Coefficients and Concentrations of Solid Metals (The Nickel-Copper System), Jpn. J. Phys., 1933, 8, p 109-113

25. P. Shewmon, Diffusion in Solids, 2nd ed., TMS, Warrendale, PA, 1998

26. T.H. Heumann and H. Mehrer, Diffusion in Metallen (Translated in Japanese by S. Fujikawa), Springer, 2005

27. H. Mehrer, Diffusion in Solids, Springer, Berlin, 2007
28. H. Mehrer, Diffusion in Solid Metals and Alloys, LandoltBornstein New Series, Vol 3-26, Springer, 1990

29. F. Sauer and V. Freise, Diffusion in Binaren Gemischen mit Volumenanderung, Z. Elektrochem., 1962, 66, p 353-363

30. P. Villars and K. Cenzual, Pearson's Crystal Data: Crystal Structure Database for Inorganic Compounds, ASM International, Materials Park, PA, 2007

31. A.S. Darling, Iridium Platinum Alloys-A Critical Review of Their Constitution and Properties, Platinum Met. Rev., 1960, 4, p 18-26

32. Y.V. Shubin, A.V. Zadesenets, A.B. Venediktov, and S.V. Korenev, Double Complex Salts $\left[\mathrm{M}\left(\mathrm{NH}_{3}\right)_{5} \mathrm{Cl}\right]\left[\mathrm{M}^{\prime} \mathrm{Br}_{4}\right]$ $\left(\mathrm{M}=\mathrm{Rh}\right.$, Ir, Co, Cr, Ru; $\left.\mathrm{M}^{\prime}=\mathrm{Pt}, \mathrm{Pd}\right)$ : Synthesis, X-Ray Diffraction Characterization, and Thermal Properties, Russ. J. Inorg. Chem., 2006, 51, p 202-209

33. W.F. Gale and T.C. Totemeier, Ed., Smithells Metals Reference Book, 8th ed., Butterworth-Heinemann, Oxford, 2004

34. M.S.A. Karunaratne and R.C. Reed, Interdiffusion of the Platinum-Group Metals in Nickel at Elevated Temperatures, Acta Mater., 2003, 51, p 2905-2919 